\title{
EDUCACIÓN EN UNA CULTURA DE LA EXCLUSIÓN, LA MARGINACIÓN Y EL RIESGO GLOBAL
}

Diego German Pérez Villamarín ${ }^{1}$

\section{Resumen}

Este artículo analiza el fenómeno educativo, desde las tendencias de la sociedad actual, en la que, a pesar de que la educación es un derecho ciudadano y una obligación de los estados, no se ha librado a las grandes masas sociales del riesgo global a la exclusión y a la marginación. Esta gran tendencia, enunciada por el sociólogo alemán Ulrich Beck y resaltada por el filósofo austriaco Ivan Illich, ofrece un particular paradigma desde donde reflexionar, evaluar y proponer un análisis y una alternativa a los riesgos sobre los que hoy se debate el mundo; es por ello que se ofrecerá el cruce, las coincidencias y las diferencias desde lo que se consideran las líneas capitales de sus propuestas que mayor repercusión

1 Doctor en Filosofía de la Pontificia Universidad Santo Tomás de Aquino -Angelicum- Roma, Italia. Posdoctor en Educación e Interculturalidad de la Universidad Santo Tomás de Bogotá. Docente e Investigador de la Facultad de Filosofía de la Universidad Santo Tomás. 
pueden tener al plantearnos problemas relacionados con la educación en el contexto intercultural, como la exclusión, la marginación y el riesgo global.

En la primera parte del trabajo, me adentro en el análisis de las estadísticas más recientes de la Unesco y la Unicef sobre la educación de los niños; luego, en los últimos informes sobre la inversión y producción de armas tanto convencionales como nucleares, así como en la amenaza permanente de los conflictos armados que azotan al mundo, y el crecimiento de la exclusión, la marginación, la desnutrición y el hambre que siguen sufriendo millones de mujeres, hombres y niños. Lo anterior no solo para alertar sino para ofrecer, a través de las reflexiones desde la perspectiva de la sociedad del riesgo global, alternativas para sobrepasar las penurias de la especie humana. Es importante, entonces, empezar por aclarar lo que significa desde esta postura la «sociedad del riesgo», para definir el concepto de riesgo y el modo como este se manifiesta en el caso particular de la educación intercultural.

\section{La sociedad del riesgo global}

Cercano a la posición de Habermas, Beck (2001) identifica una sociedad en la que se diluye el pensamiento fuerte y se produce un alejamiento del compromiso social, una sociedad en la que se da una desregulación absoluta de las relaciones, una sociedad en la el poder político es captado por el poder económico, estableciendo una fuente de incertidumbre, inseguridad y riesgos. La sociedad posmoderna asume una carga de riesgo en su propia identidad que encierra una grave contradicción: el peligro de la supervivencia de la especie. La rentabilidad del sistema corre el riesgo de la incertidumbre; no asume como punto de partida la seguridad y la sostenibilidad en términos ecológicos, sino que conoce que existen márgenes de peligrosidad para la especie, cuya preocupación no es paradójicamente prioritaria por encontrarse en un sistema guiado por la obtención de beneficios y una representación retórica de la racionalidad. En todo caso, Beck (2006 p. 115 ) insistirá en que la sociedad del riesgo es aquella en la que los riesgos no pueden abordarse y resolverse según los parámetros y estándares institucionalizados de la sociedad industrial, en la que el concepto de seguridad ha quedado desbordado por los peligros que genera.

Beck (2011) reconoce que el debate modernidad/posmodernidad se ha vuelto fatigoso, por lo que la idea de modernidad reflexiva nos libra del anquilosamiento de estos debates. Por otra parte, Beck identifica un cambio de estatus de la tradición, caracterizada como 
detradicionalización; asimismo, resalta la preocupación por cuestiones ecológicas, haciendo énfasis en que el medio ambiente no es ya un hecho externo a la vida social humana sino que está totalmente impregnado y reordenado por ella (pp. 223-224).

De otra parte, Beck considera que el conocimiento humano se hace cada día más reflexivo, el futuro se parece cada vez menos al pasado y en ciertos aspectos básicos se ha hecho muy amenazador; de ahí que la noción de riesgo sea esencial para la cultura moderna. Pero a diferencia de la historicidad que media en la edad moderna, en la sociedad del riesgo el futuro, antes de ser un glorioso amanecer para la humanidad, se convierte en un futuro potencial en el que se crean nuevas áreas de imprevisibilidad, en las que las instituciones y la sociedad industrial, en general, fracasan víctimas de su propio éxito; de modo que antes de construir futuro, nos estamos quedando sin él.

En el siguiente cuadro se establecen algunas de las categorías con las que Beck identifica y sinteza de modo general la evolución de la sociedad del riesgo. A partir de estas categorías se evalúan las cifras y estadísticas referidas más adelante. 


\begin{tabular}{|c|c|}
\hline Primera modernidad & Segunda modernidad \\
\hline $\begin{array}{l}\text { - Industrializada } \\
\text { - Controlada y segura }\end{array}$ & $\begin{array}{l}\text { - Modernidad radicalizada } \\
\text { - Impredecible }\end{array}$ \\
\hline $\begin{array}{l}\text { - Estados nación } \\
\text { - Territorios bien definidos } \\
\text { - Pautas colectivas de vida } \\
\text { - Progreso y controlabilidad } \\
\text { - Pleno empleo y explotación de la naturaleza }\end{array}$ & $\begin{array}{l}\text { - Globalización } \\
\text { - Individualización } \\
\text { - Revolución de géneros } \\
\text { - Riesgos globales } \\
\text { - Subempleo y catástrofes causadas por el } \\
\text { hombre }\end{array}$ \\
\hline $\begin{array}{l}\text { Estos procesos radicalizados constituyen } \\
\text { la modernización reflexiva. }\end{array}$ & $\begin{array}{l}\text { Colapso de la idea de controlabilidad, } \\
\text { certidumbre o seguridad. }\end{array}$ \\
\hline \multicolumn{2}{|c|}{$\begin{array}{l}\text { Está constituyéndose un nuevo tipo de capitalismo, un nuevo tipo de economía, un nuevo tipo } \\
\text { de sociedad, un nuevo tipo de vida personal. Por lo tanto sociológica, política y educativamente, } \\
\text { necesitamos un cambio de paradigma en el que se revaloren sus aspectos centrales. }\end{array}$} \\
\hline En el lado positivo & En el aspecto negativo \\
\hline $\begin{array}{l}\text { - Sociedades multireligiosas, multiétnicas y } \\
\text { multiculturales. } \\
\text { - Modelos interculturales, multiétnicos y } \\
\text { multiculturales. Tolerancia de la diferencia } \\
\text { cultural. } \\
\text { - El pluralismo legal que se observa en muchos } \\
\text { países. } \\
\text { - La multiplicación de las soberanías. }\end{array}$ & $\begin{array}{l}\text { - Extensión de la informalidad económica. } \\
\text { - La flexibilización del trabajo y de las } \\
\text { relaciones laborales. } \\
\text { - La pérdida de legitimidad del estado. } \\
\text { - Crecimiento del empleo y el subempleo. } \\
\text { - Intervenciones cada vez mayores de las } \\
\text { multinacionales. } \\
\text { - Aumento de la violencia y el crimen. }\end{array}$ \\
\hline
\end{tabular}

\section{Gasto mundial en armas y sociedad reflexiva}

Una vez caracterizada la sociedad del riesgo, a continuación se verá cómo una vez más el riesgo se hace inminente ante las cifrass mundiales sobre la inversión del gasto mundial en armas tanto convencionales como nucleares, mostrándonos que de un mundo de enemigos producto de la Guerra Fría, pasamos a un mundo de peligros y riesgos ecológicos, nucleares, armamentistas, en el que se pone en riesgo la supervivencia de la especie humana. Veamos, por ejemplo, en estadísticas recientes, el cálculo del gasto militar mundial para 2011 que ha sido de 1738 billones de dólares, cifra que representa el 2,5 \% del producto interno bruto mundial (249 dólares por persona). En comparación con 
el total del 2010, el gasto militar permaneció prácticamente invariable en términos reales. No obstante, todavía es muy pronto para decir si eso significa que el gasto militar mundial finalmente haya tocado techo.

La causa principal de que se haya detenido el aumento del gasto militar, según lo muestra la tabla siguiente, han sido las políticas económicas adoptadas en la mayoría de países occidentales a raíz de la crisis financiera y económica mundial que empezó en 2008. Esas políticas dieron prioridad a la reducción rápida de los déficits presupuestarios que aumentaron drásticamente al estallar la crisis (Sipri, 2012, p. 10).

Tabla 1. Gasto militar mundial, 2011

\begin{tabular}{lcc}
\hline \multicolumn{1}{c}{ Región } & Gasto (miles de millones de \$) & Cambio (\%) \\
\hline África & 34,3 & 8,6 \\
\hline Norte de África & 3,9 & 25,0 \\
\hline África Subsahariana & 20,4 & $-0,1$ \\
\hline América & 809,0 & $-1,4$ \\
\hline Centroamérica y Caribe & 7,0 & 2,7 \\
\hline Norteamérica & 736,0 & $-1,2$ \\
\hline Sudamérica & 66,0 & $-3,9$ \\
\hline Asia y Oceanía & 364,0 & 2,2 \\
\hline Asia central y sur asiático & 61,7 & $-2,7$ \\
\hline Este asiático & 243,0 & 4,1 \\
\hline Oceanía & 28,6 & $-1,2$ \\
\hline Sudeste asiático & 31,0 & 2,7 \\
\hline Europa & 407,0 & 0,2 \\
\hline Europa oriental & 80,5 & 10,0 \\
\hline Occidental y central & 326,0 & $-1,9$ \\
\hline Oriente próximo & 123,0 & 4,6 \\
\hline Total mundial & $\mathbf{1 . 7 3 8 , 0}$ & $\mathbf{0 , 3}$ \\
\hline Cifras de gasto en dólares corrientes (2011). & & \\
\hline
\end{tabular}

Fuente: Sipri yearbook 2012 
A inicios de 2012, ocho estados poseían aproximadamente 4.400 armas nucleares operativas, de las cuales casi 2.000 se mantienen en un estado de alerta operativa alta. Si se contabilizan todas las ojivas nucleares -ojivas operativas, armas de reposición, las que están en almacenamiento activo e inactivo y ojivas intactas programadas para su desmantelamiento-, Estados Unidos, Rusia, el Reino Unido, Francia, China, la India, el Pakistán e Israel poseen en conjunto un total aproximado de 19.00o armas nucleares.

Tabla 2. Fuerzas nucleares en el mundo, 2012

\begin{tabular}{lccc}
\hline \multicolumn{1}{c}{ País } & Ojivas desplazadas & Otras ojivas & Inventario \\
\hline E.E.U.U. & 2.150 & 5.850 & 8.000 \\
\hline Rusia & 1.800 & 8.200 & 10.000 \\
\hline Reino Unido & 160 & 65 & 225 \\
\hline Francia & 290 & 10 & -300 \\
\hline China & 200 &,,, & -240 \\
\hline India &.. & $80-100$ & $80-100$ \\
\hline Pakistán &, & $90-110$ & $90-110$ \\
\hline Israel & & -80 & -80 \\
\hline Corea del Norte &,, &,, & ?? \\
\hline Total & -4.400 & -14.600 & -19.000 \\
\hline
\end{tabular}

Todas las estimaciones son aproximadas y correspondientes a enero de 2012

Fuente: Sipri yearbook 2012.

Estas cifras, que no solo quieren ser estadística, manifiestan que con la explosión de solo una parte mínima de este arsenal nuclear un desastre cósmico puede suceder en este mismo instante. Asimismo, dejan ver que los silos de la muerte de los países más ricos están malbaratando las posibilidades de una vida mejor para todos.

A pesar de estas cifras tan alarmantes, en la sociedad reflexiva se prevé que es posible que uno de los aspectos de mayor trascendencia sea que muchos de los problemas de seguridad más importantes de los próximos años no se presten fácilmente a las soluciones militares tradicionales. En cambio, lo que será necesario es una integración innovadora de la diplomacia preventiva, de las tecnologías preventivas, de alerta temprana de las asociaciones transnacionales de cooperación (Sipri, 2012). 
Estas cifras que hablan por sí solas indican el riesgo al que está sometida la humanidad, por lo cual se hace necesario un nuevo paradigma social-científico-técnico-educativo.

La propuesta de Beck (2011) sobre la Modernización Reflexiva (MR) significa la posibilidad de una (auto)destrucción creativa de toda época, la de la sociedad industrial. La MR significa la desvinculación y luego la revinculación de formas de vida industriales por otro tipo de modernidad. En esta nueva etapa en la que el progreso puede convertirse en autodestrucción, en la que un tipo de modernidad socava y transforma otra en virtud de su dinámica independiente y de sus éxitos, la sociedad industrial se está deslizando a tierra de nadie. Se están originando líneas de conflicto respecto al qué y al cómo del progreso, líneas que están adquiriendo la capacidad de organizarse y constituir coaliciones. Se trata del retorno de la incertidumbre, no hay ningún sujeto claramente definible, ni la clase obrera, ni las mujeres, ni lo público, ni lo privado. En torno a la incertidumbre, dados los efectos colaterales no percibidos, sobreviene la crisis de la sociedad industrial. Sus consecuencias se presentan únicamente dentro de los conceptos y dentro de la perspectiva de la sociedad del riesgo. Se hace necesaria una autodeterminación reflexiva. En la sociedad del riesgo, la amenaza precisa la autorreflexión sobre los fundamentos de la cohesión social y el examen de las convicciones y fundamentos dominantes de la «racionalidad». En el autoconcepto de la sociedad del riesgo, la sociedad deviene reflexiva, se convierte en un tema y en un problema para sí misma. La categoría del riesgo es postradicional, el riesgo surge a partir del triunfo de la razón; no obstante, la razón es también la base de una autocrítica pluralista de la sociedad. La sociedad del riesgo es tendencialmente una sociedad autocrítica en la que ya no hay no-participantes (Beck. 1997, p. 22).

\section{¿Qué quiere decir riesgo?}

Riesgo es el enfoque moderno de la previsión y control de las consecuencias futuras de la acción humana; las diversas consecuencias no deseables de la modernización radicalizada. El riesgo es un intento (institucionalizado) de colonizar el futuro, un mapa cognitivo.

Los riesgos son híbridos creados por el hombre. Incluyen y combinan la política, la ética, las matemáticas, los medios de comunicación de masas, las tecnologías, las definiciones y percepciones culturales y, lo más importante de todo, no se pueden separar estos aspectos y realidades si se quiere entender la dinámica cultural y política de la sociedad del riesgo global, nutrida por las ciencias de la complejidad. Por tanto, «la del 
riesgo», no es únicamente una noción que se utilice como tema central en disciplinas muy diversas, sino que es el modo en que la «sociedad hibrida» observa, describe, valora y critica su propia naturaleza hibrida. (Beck, 2006, p. 232).

Una sociedad que se percibe a sí misma como sociedad del riesgo deviene reflexiva, es decir, los fundamentos de su actividad y sus objetivos se convierten en objeto de controversias científicas y políticas públicas.

El riesgo puede, asimismo, ser entendido en los términos de Illich (2006):

Desde las amenazas que por ejemplo la medicina actual representa para la salud de las poblaciones, la amenaza que el volumen y la intensidad del tráfico representa para la movilidad, la amenaza que la educación y los medios masivos de comunicación representan para el aprendizaje, y la amenaza que la urbanización representa para la habilidad de construir una morada (p. 538).

La escuela misma, en este contexto del riesgo, ya no es un buen instrumento de educación, ni el automóvil un buen instrumento de transporte, ni la línea de montaje un buen instrumento de producción; la escuela produce males, la velocidad devora el tiempo y la medicina la salud.

En esta línea, Illich insiste no solo en una radical inversión de las instituciones industriales, sino en su eliminación, pues únicamente invirtiendo la lógica de la institución se hace posible revertir el movimiento. Por esta inversión, ni la industria, ni la burocracia serán destruidas sino eliminadas como impedimentos a otros modos de producción. Mientras no se inviertan las instituciones imperantes y no se sustituya la instrumentación industrial por herramientas convivenciales, el ideal propuesto por la tradición socialista no se hará realidad.

Esta crisis planetaria de las instituciones, planteada por Beck, puede ser entendida por Illich como la que nos puede hacer llegar a un nuevo estado de conciencia, que afecte a la naturaleza de la herramienta y a la acción a seguir para que la mayoría tome el control. Frente a la amenaza de un apocalipsis tecnocrático, ecológico, armamentista y nuclear, Illich propone una sociedad convivencial, la cual descansará sobre sus contratos sociales que garanticen a cada uno el mayor y mejor libre acceso a las herramientas de la comunidad, con la condición de no lesionar una igual libertad de acceso al otro (Illich, 2006, pp. 384-386).

En la era industrial (primera modernización) el núcleo familiar era la célula social, la matriz cultural y laboral, la estructura de la socialización primaria, la unidad económica. 
Al pasar a la «segunda modernización», ese núcleo se rompe y la dinámica de las sociedades impregnada por los valores mercantiles impulsa la vida hacia la individualización de las estructuras, hacia situaciones donde se acentúa la incertidumbre del individuo, dadas las condiciones precarias del trabajo, el escepticismo, la marginación y el desarraigo. El individualismo aparece como un efecto esterilizador del neoliberalismo económico, un vacío institucional que se manifiesta en la crisis de los sindicatos, de los partidos, de los mecanismos de representación y de gestión pública

La sociedad industrial socava sus propios fundamentos. Lo que se afirma es que el dinamismo industrial de alta velocidad se está deslizando hacia una nueva sociedad sin la explosión primigenia de una revolución, dejando a un lado los debates políticos y las decisiones de parlamentos y gobiernos. Lo anterior significa, entonces, una radicalización de la modernidad que quiebra las premisas y contornos de la sociedad industrial y abre vías a una modernidad distinta.

La idea de arte de la política que Beck proyecta junto con Giddens bajo el enunciado de la tercera vía se basa en la necesidad no tanto de redactar nuevas normas como de adaptarlas a la realidad social, a las necesidades del momento, al patrimonio de la sociedad civil. En esta misma línea, Beck, convencido de la necesidad de una cooperación supranacional como instrumento de refuerzo del desarrollo y la estabilidad local; frente a los valores del viejo estado nacional, fuerte y jerarquizado, describe el Estado cosmopolita, autónomo ciertamente, pero plenamente abierto a la cooperación y a la cohesión internacional, que, manteniendo la diversidad, amortigüa las tensiones entre las diferencias (Beck, 1997)

Beck sostiene que la transición de una época social a otra puede tener lugar de forma no pretendida y apocalíptica, la nueva sociedad no siempre nace con dolor. Una sociedad nueva sustituirá a la antigua sin que exista una revolución. No es la creciente pobreza, sino la riqueza y la pérdida de su rival oriental lo que produce un tiempo axial en los tipos de problemas; un vigoroso crecimiento económico, una rápida tecnificación y una elevada seguridad laboral son los que pueden desencadenar la tormenta en la que la sociedad industrial navegará o será arrastrada hacia una nueva época.

Esta modernización de la modernización es un fenómeno de gran importancia que requiere la máxima atención. La Modernidad Reflexiva puede tener consecuencias opuestas: nacionalismo, pobreza masiva, fundamentalismos religiosos, crisis económicas, crisis ecológicas, posiblemente guerras y revoluciones, estados de emergencia provocados por grandes accidentes catastróficos; es decir el dinamismo de la sociedad del riesgo en el sentido más estricto. 
Ante la insolencia de la sociedad industrial, aparece la nueva sociedad, la nueva sociología, la de la sociedad del riesgo. Se designa así una fase del desarrollo de la sociedad moderna en la que los riesgos sociales, políticos, económicos e individuales tienden cada vez más a escapar a las instituciones de control y protección de la sociedad industrial.

En la sociedad del riesgo se establecen dos fases: En la primera, los efectos y autoamenazas son producidos en forma sistemática pero no se convierten en debate público o en el centro de los debates políticos, lo que permite la multiplicación y legitimación de las amenazas producidas por la toma de decisiones como riesgos residuales. En una segunda fase, los peligros de la sociedad industrial no se pueden controlar, las características de la sociedad industrial se hacen social y políticamente problemáticas. Los dinamismos que derivan de la sociedad del riesgo ciernen sobre las organizaciones de interés, el sistema judicial y educativo, y la política (Beck, ¿año?, p.18).

En este orden de ideas, cabe preguntarse: ¿qué desafíos educativos están vinculados a los desafíos de la sociedad del riesgo y qué retos son concebibles?

\section{Sociedades iatrogénicas, educación excluyente}

La situación resulta iatrogénica (los sistemas que normalmente son incorporados para curar o sanar las enfermedades de un sistema, resultan ellos mismos patógenos, es decir devorados por su propia creación). El término, acuñado por Illich, indica cómo la contraproductividad específica afecta a todas las relaciones sociales: así, la aceleración del tráfico, consumidora del tiempo; las comunicaciones ruidosas y confusas; la educación que entrena cada vez más gente para niveles de competencia técnica y formas especializadas de incompetencia cada vez más altas (2006, p 538). En cada caso, un gran sector institucional ha apartado a la sociedad del propósito específico para el cual dicho sector fue creado y técnicamente instrumentado. La iatrogénesis muestra ser más una paradoja que una contraproductividad específica, como un indicador social negativo que puede observarse en todos los sectores principales de la sociedad industrial. En un principio se aplica un nuevo conocimiento a la solución de un problema claramente definido y los criterios científicos permiten medir los beneficios obtenidos en eficiencia; pero, en seguida, el progreso obtenido se convierte en medio para explotar al conjunto social, para ponerlo al servicio de los valores que una élite especializada, garante de su propio valor, determina y revisa constantemente (Illich, 2008, p. 381). 
En esta misma línea, Illich (2006) propone que la educación pública se beneficiaría con la desescolarización (p.192) y muestra lo que podría significar la desescolarización en una sociedad escolarizada. Para ello, menciona como uno de sus principales argumentos que el fanatismo a favor de la escuela hace posible la explotación en doble sentido: por una parte, el aumento de fondos públicos para la asignación de recursos para la educación de unos pocos, y por otra, la explotación a través del control social por parte de la mayoría (p 196). Illich sostiene, además, que ni en Norteamérica ni en América Latina logran los pobres igualdad a partir de escuelas obligatorias, pero en ambas partes la sola existencia de la escuela desanima al pobre y lo invalida para asir el control de su propio aprendizaje. En todo el mundo, dice Illich, la educación tiene un efecto antieducacional sobre la sociedad; esta visión parece estar confirmada por el último informe sobre educación de la UnescoUnicef, en el que se alerta que 22.1 millones de niños están fuera de las aulas.

El gasto derivado de la repetición de cursos escolares en América Latina y el Caribe supera los 19.980 millones de dólares. Asimismo, el informe cuestiona el hecho de que el sobrecosto que significa el fracaso escolar, manifestado en la repetición de años escolares, es equivalente a tres veces el valor de las metas de cobertura universal desde el nivel inicial (preescolar o jardín) hasta el secundario inferior. El estudio de Unicef, en el que traza el panorama educativo de la región, demuestra que el dinero que se invierte en los estudiantes que deben volver a cursar un año escolar representa el o.34 \% del PIB de los países latinoamericanos y caribeños y un $7 \%$ del gasto educativo regional. Los gastos son de tan altas proporciones debido a que alrededor del $13 \%$ de los alumnos matriculados en la educación inicial, primaria y secundaria está repitiendo un curso.

Los sobrecostos improductivos para el sistema de la región (que invierte el $4.5 \%$ de su PIB en educación, frente al $\mathbf{1 2 . 4}$ \% de los países más desarrollados) han incrementado alarmantemente el costo de producir un egresado, por el que se paga dos o más veces para que consiga un mismo grado. Los estudiantes que repiten algún curso hacen parte de los 15.6 millones de niños y adolescentes que asisten a la escuela arrastrando algún tipo de fracaso escolar, con lo cual quedan en riesgo de no culminar sus estudios. Esto ha sido determinante también para que actualmente el 22 por ciento de los estudiantes que deberían estar cursando la secundaria no hayan finalizado la primaria, debido de igual forma al ingreso tardío al sistema educativo (Unesco, 2012). 


\section{Cinco dimensiones de exclusión}

Según el informe «Completar la escuela. Un derecho para crecer, un deber para compartir», presentado en agosto de 2012 por el Instituto de Estadísitica (UIS) de la Unesco y la Unicef, en América Latina y el Caribe hay aproximadamente 117 millones de niños, niñas y adolescentes en edad de asistir a la educación inicial, primaria y secundaria básica. De ellos, 6.5 millones no asisten a las escuela y 15.6 millones asisten arrastrando fracasos y dando graves señales de desigualdad (dos o más años de desfase grado-edad o rezago escolar).

En el marco del informe se determinan cinco dimensiones de exclusión, es decir, cinco posibilidades de estar hoy o mañana fuera de la escuela y del mapa del recorrido escolar:

\section{Tabla 3. Dimensiones de exclusión}

\begin{tabular}{|c|c|c|c|}
\hline 21.1 Millón & & & \\
\hline $\begin{array}{l}\text { Niños en edad de asistir a la educación inicial que } \\
\text { la escuela inicial o primaria }\end{array}$ & no están en & $1.7 \mathrm{r}$ & es $(*)$ \\
\hline $\begin{array}{l}\text { Niños en edad de asistir a la escuela primaria que } \\
\text { la escuela primaria ni en la secundaria básica. De } \\
\text { escuela inicial con rezago }{ }^{(*)} 1.4 \text { millones }\end{array}$ & $\begin{array}{l}\text { no están en } \\
\text { os cuales, en }\end{array}$ & 2.91 & es $(*)$ \\
\hline $\begin{array}{l}\text { Niños-Adolescentes en edad de asistir a la educac } \\
\text { secundaria básica que no están en la escuela prim } \\
\text { secundaria }\end{array}$ & on & $1.9 \mathrm{r}$ & es (*) \\
\hline $\begin{array}{l}\text { Niños que están en la escuela primaria pero que } \\
\text { están en riesgo de abandonarla }\end{array}$ & 9.2 millones & $\star *)$ & $\begin{array}{l}\text { Otros } 14 \cdot 7 \\
\text { millones }\end{array}$ \\
\hline $\begin{array}{l}\text { Niños-adolescentes que están en la escuela } \\
\text { secundaria básica pero que están en riesgo de } \\
\text { abandonarla. }\end{array}$ & 6.4 millones & $* *)$ & $\begin{array}{l}\text { Otros } 8.1 \\
\text { millones }\end{array}$ \\
\hline
\end{tabular}

Fuente: ${ }^{*}$ ) estimaciones del Instituto de Estadística de la Unesco (UIS) para la región, correspondientes al año 2009; (**) estimaciones propias en Base a datos del Instituto de Estadística de la Unesco (UIS), correspondientes a 2008.

Los resultados no pueden ser menos procupantees; sin embargo los esfuerzos continúan, pero las cifras siguen siendo alarmantes cuando se evidencia la inversión mundial en armas. 
Respecto a la asistencia infantil, Unicef calculó, en 1981, un programa para resolver los problemas esenciales de los 500 millones de niños más pobres del mundo. Este programa comprendía la asistencia sanitaria de base, la educación elemental, la mejora de las condiciones higiénicas, del abastecimiento de agua potable y alimentación. Todo esto parecía un sueño imposible de 100.000 millones de dólares; sin embargo, ese es apenas el costo de cien bombarderos B- B1 y de menos de siete mil cohetes Crucero, en cuya producción ha de invertir el gobierno de Estados Unidos 21.200 millones de dólares.

Sobre la educación, por ejemplo, según García Márquez (2010), con solo dos submarinos atómicos Trident, de los veinticinco que planeaba fabricar el gobierno de los Estados Unidos, o con una cantidad similar de los submarinos Tifón que está construyendo la Unión Soviética, podría intentarse la alfabetización mundial. Por otra parte, la construcción de las escuelas y la calificación de los maestros que harán falta para el Tercer Mundo para atender las demandas adicionales de la educación en los diez años por venir, podrían pagarse con el costo de 245 cohetes Tridente II, y aún quedarían sobrando 419 cohetes para el mismo incremento de la educación en los quince años siguientes. Claro es pues que la carrera de las armas va en sentido contrario a la de la inteligencia (García Márquez, 2010, pp. 45-48)

La mayoría de las personas, dice Illich, considera los fracasos de la escuela como una prueba de que la educación es una tarea muy costosa, muy compleja, siempre arcana y frecuentemente casi imposible (2006, p. 197); se evidencia así mismo que tanto en Estados Unidos como en América Latina los costes de la educación per cápita han aumentado casi con igual rapidez que el costo del tratamiento médico; la escuela se apropia del dinero de los hombres y de la buena voluntad disponibles para la educación, pero este tratamiento más completo impartido por doctores y maestros ha mostrado resultados en continua decadencia.

En su diagnóstico, Illich asevera sin temor alguno que la escalada de las escuelas es muy destructiva, aunque de manera menos visible que el de las armas; en todas partes los gastos en la escuela se quedan cada vez más cortos frente a las expectativas de padres, maestros y alumnos, de modo que una escolaridad pareja no solo es impracticable sino que resulta económicamente absurda, intelectualmente castrante, socialmente polarizante y destruye el sistema político que la promueve (2006, p. 199). 


\section{Conclusión}

La imposibilidad de la reconstrucción social, técnica, política y educativa radica en las estructuras que detentan el poder, de ahí que los cambios deban invertir radicalmente las instituciones industriales y reconstruir la sociedad totalmente. Por esta inversión radical, la ciencia y la tecnología modernas no serán aniquiladas, sino que dotarán a la actividad humana de una eficacia sin precedentes. Por esta inversión ni la industria ni la burocracia serán destruidas, sino eliminadas como obstáculos de otros modos de producción. La convivencialidad será restaurada en el centro mismo de los sistemas políticos que protegen, garantizan y refuerzan el ejercicio óptimo del recurso que mejor repartido está en el mundo: la energía personal que controla la persona. Hay que trasladarse de la productividad a la convivencialidad, sustituir un valor técnico por un valor ético, un valor material por un valor realizado.

Saludamos la crisis declarada de las instituciones dominantes como el amanecer de una liberación revolucionaria que nos emancipará de aquellas instancias que mutilan la libertad elemental del ser humano, con el solo fin de atosigar cada vez a más usuarios. Esta crisis planetaria de las instituciones nos puede hacer llegar a un nuevo estado de conciencia que afecte la naturaleza de la herramienta y a la acción a seguir, para que la mayoría tome el control.

Se trata, entonces, de reivindicar la libertad y la dignidad del ser humano. Pero, antes que nada, de fundamentar la sociedad convivencial bajo la égida de tres valores: la sobrevivencia, la equidad y la autonomía creadora. Cada uno de estos valores es una ficha importante en el andamiaje de la sociedad y se interrelacionan entre sí, de tal modo que la sobrevivencia da lugar a la equidad, y estas dos, a su vez, constituyen a la autonomía, que da lugar al trabajo convivencial.

Se propone la construcción de una sociedad posindustrial en la que el ejercicio de la creatividad de una persona no imponga jamás a otra un trabajo, un conocimiento o consumo obligatorio. En la era de la tecnología científica, solamente una estructura convivencial de la herramienta puede conjugar la supervivencia y la equidad. La equidad exige que, a un tiempo, se compartan el poder y el haber. Una estructura convivencial de la herramienta hace realizable la equidad y practicable la justicia; ella constituye la única garantía de supervivencia.

Por otra parte, las estadísticas muestran la desproporción entre el gasto militar mundial y el gasto mundial en educación; la racionalidad armamentista en total desacuerdo con la necesidad de educación es arbitraria e intolerante, pero tampoco deja 
de escandalizar que la escolarización del mundo resulta casi insostenible: en principio los estados no alcanzarían sin grandes sacrificios a brindar educación a los ciudadanos (niños y jóvenes), por eso la propuesta de Illich podría orientar nuevos modos pedagógicos que empiecen por reconocer las habilidades personales, impulsando el uso de la energía creadora de cada individuo hacia el cual debe orientarse el gasto público.

Si para Beck la sociedad posindustrial vendrá sin hacerse sentir, para Illich, por su parte, el tránsito de un modo de producción industrial a un modo de producción convivencial será dramático y exigirá el sacrificio de todos los actores de la sociedad, pobres y ricos, en tanto la convivencialidad será fruto de toda una revolución en la que la idea es renunciar a la sobrepoblación, la sobreabundancia y al superpoder, sin importar si se trata de grupos o individuos. En esa medida, la convivencialidad no tiene precio, pero se debe saber muy bien lo que costará desprenderse del modelo actual. Para Illich, la convivencialidad será el producto de una ascesis personal, quizá utópica, quizá real en la medida en que al hombre llegue a la conviviencialidad como un reto de su quehacer; pues en ella el hombre reencontrará la alegría de la sobriedad y de la austeridad, reaprendiendo a depender del otro, en vez de convertirse en esclavo de la energía y de la burocracia todopoderosa.

\section{Referencias}

Giddens A, Bauman Z. Luhmann N. Beck U. (2011) Las consecuencias perversas de la modernidad. Anthropos.

García Márquez, G. ( 2010 ) Yo no vengo a decir un discurso. Bogotá D. C., Colombia: Random House Mondadori, S.A.

Illich, I. (2006). Obras reunidas I. México: Fondo de Cultura Económica

Illich, I. (2008). Obras reunidas II. México: Fondo de Cultura Económica.

Instituto Internacional de Estocolmo para la investigación de la paz SIPRIYearbook. (2012). Armaments, Disarmament and International Security. Recuperado de: www.fundacionperlapau.org

Ulrich, B. (2006). La sociedad del riesgo global. Traducción de Jesús Alborés Rey. Madrid, España: Siglo XXI Editores.

Ulirich, B. Gidens, A. y Lash, S. (1997). Modernización reflexiva, política, tradición y estética en el orden social moderno. Traducción de Juan Ignacio Luca de Tena. Madrid: Alianza Editorial S.A

Unesco - Unicef. (2012). Informe sobre la educación en América Latina y el Caribe. Recuperado de: www.unesco.org. 
Este texto se editó en Ediciones USTA, Departamento Editorial de la Universidad Santo Tomás. Se usó papel XXXX para cubierta y papel XXXX para páginas internas. Fuente: Eidetic Neo OT. Impreso por XXXX.

2016 
Sabido es que América Latina está dominada por las diferencias. Por ello, no resulta extraño que la multiculturalidad y la interculturalidad sean puntos fundamentales, tal vez los más importantes, de la agenda académica y social de muchas instituciones académicas en la actualidad. Una pregunta nos sale al paso de inmediato: ¿cómo pensar a América Latina desde perspectiva intercultural? Para responder a este interrogante, cada uno de los trabajos aquí compilados pone de manifiesto que el conocimiento puede promover vinculaciones sociales y compromisos cívicos, ya que el conocimiento no habla de nosotros, sino que habla con nosotros y cada uno se siente enunciado por él. 